\title{
Symmetries for standard model alignment in multi-Higgs doublet models
}

\author{
Apostolos Pilaftsis ${ }^{*}$ \\ Consortium for Fundamental Physics, School of Physics and Astronomy, University of Manchester, \\ Manchester M13 9PL, United Kingdom and Theory Division, CERN, CH-1211 Geneva 23, Switzerland
}

(Received 8 February 2016; published 7 April 2016)

\begin{abstract}
We derive the complete set of continuous maximal symmetries for standard model (SM) alignment that may occur in the tree-level scalar potential of multi-Higgs doublet models, with $n>2$ Higgs doublets. Our results generalize the symmetries of SM alignment, without decoupling of large mass scales or fine-tuning, previously obtained in the context of two-Higgs doublet models.
\end{abstract}

DOI: 10.1103/PhysRevD.93.075012

\section{INTRODUCTION}

As more and more data are being collected at the CERN Large Hadron Collider (LHC), it becomes even more apparent from global analyses [1-5] that the observed Higgs boson $h$, with a mass $M_{h} \approx 125 \mathrm{GeV}$, interacts with the gauge bosons with coupling strengths that are very close to those predicted by the standard model (SM) [6]. The constraints deduced from the strengths of these Higgs couplings, primarily to $W^{ \pm}$and $Z$ bosons, put severe limits on the actual form of a possible heavy scalar sector in the observable sub- $\mathrm{TeV}$ range, and so on the model structure of new physics to be anticipated at the LHC.

If there are additional heavy scalars in the theory, as predicted in two-Higgs doublet models (2HDMs) [7-9] or $n$ HDMs, with $n>2$ Higgs doublets [10-15], there are two main strategies that are followed in the literature to avoid too large mixings of the heavy scalars to the SM Higgs boson $h$. The first one is known as the decoupling limit [16-18], in which the heavy scalars are made very heavy, such that they effectively decouple from the low-energy SM sector altogether. The second strategy, which is of interest to us, is a bit more optimistic, as it leaves open the possibility to directly probe the heavy scalar sector. It assumes that the new scalars are not very heavy after all, e.g. they have masses in the sub-TeV range, but the theoretical parameters are arranged in such a way that the new scalars do not mix significantly with $h$. As a consequence, the $h W^{+} W^{-}$and $h Z Z$ couplings retain their SM values, to a very good approximation. In the $2 \mathrm{HDM}$, such parameter arrangement leads to a sort of alignment between the $2 \times 2$ charge parity $(C P)$-odd scalar mass matrix $\mathcal{M}_{P}^{2}$ and the $2 \times 2 C P$-even scalar mass matrix $\mathcal{M}_{S}^{2}$ in the $C P$-conserving limit of the theory. In the so-called SM-alignment limit [19-26], the two mass matrices $\mathcal{M}_{P}^{2}$ and $\mathcal{M}_{S}^{2}$ get diagonalized by means

*apostolos.pilaftsis@manchester.ac.uk

Published by the American Physical Society under the terms of the Creative Commons Attribution 3.0 License. Further distribution of this work must maintain attribution to the author(s) and the published article's title, journal citation, and DOI. of the same mixing angle, e.g. $\beta$, where $\tan \beta=v_{2} / v_{1}$ is the ratio of the vacuum expectation values (VEVs) of the two Higgs doublets $\Phi_{1}$ and $\Phi_{2}$, i.e. $\left\langle\Phi_{1}\right\rangle=v_{1} / \sqrt{2}$ and $\left\langle\Phi_{2}\right\rangle=v_{2} / \sqrt{2}$.

An unpleasant aspect of achieving SM alignment without decoupling of mass scales is the degree of fine-tuning that is frequently required among the theoretical parameters. However, a recent study has shown [24] that the phenomenologically desirable SM alignment in the 2HDM may be realized upon the imposition of symmetries on its scalar potential. In particular, three maximal symmetries, softly broken possibly by bilinear mass terms, have been identified for which the tree-level scalar potential of the 2HDM exhibits exact SM alignment. We therefore refer to this mechanism as the natural alignment mechanism. We briefly review the basic features of natural alignment in the $2 \mathrm{HDM}$ in Sec. II.

In this paper we extend the results, previously obtained in the context of 2HDMs, and derive the complete set of symmetries for SM alignment that may take place in multi-HDMs, with more than two Higgs doublets. In $n$ HDMs, with $n>2$ Higgs doublets, the task of identifying all SM-alignment symmetries becomes more laborious. An $n$ HDM may have a number $m$ of inert Higgs doublets, which do not participate in the mechanism of electroweak symmetry breaking (EWSB) because of some unbroken symmetry, such as a $Z_{2}$ symmetry [27-30], resulting in the vanishing of VEVs for all inert Higgs doublets. Nevertheless, the key observation to be made here is that SM alignment is naturally achieved in multiHDMs, if the dimension- 4 part of the scalar potential $V$ containing the quartic couplings of the EWSB scalars is invariant under rotations that diagonalize their corresponding squared mass matrix of dimension 2 in $V$. To put it simply, the quartic couplings involving only EWSB scalars must be invariant under SM-alignment-symmetry transformations which include the so-called Higgs basis [16] in which the bilinear mass matrix is diagonal. Our approach to deriving the symmetries for SM alignment in multiHDMs is presented in Sec. III. 
The paper has the following structure. After this introductory section, Sec. II briefly reviews the basic features of natural alignment in the 2HDM. After having gained useful insight from the 2HDM case, we derive in Sec. III the complete set of symmetries for SM alignment that may occur in the tree-level scalar potential of multi-HDMs. Finally, the key findings of our study are summarized in Sec. IV.

\section{NATURAL ALIGNMENT IN THE 2HDM}

In this section we briefly review the SM-alignment limit in the 2HDM and the symmetries that naturally enforce this limit. More details may be found in [24].

Let us start our discussion by writing down the tree-level scalar potential $V$ of the 2HDM, expressed in terms of the $\mathrm{SU}(2)_{L}$ Higgs doublets $\Phi_{1}$ and $\Phi_{2}$ with hypercharges $Y_{\Phi_{1,2}}=1$,

$$
\begin{aligned}
V= & -\mu_{1}^{2}\left(\Phi_{1}^{\dagger} \Phi_{1}\right)-\mu_{2}^{2}\left(\Phi_{2}^{\dagger} \Phi_{2}\right)-m_{12}^{2}\left(\Phi_{1}^{\dagger} \Phi_{2}\right)-m_{12}^{* 2}\left(\Phi_{2}^{\dagger} \Phi_{1}\right) \\
& +\lambda_{1}\left(\Phi_{1}^{\dagger} \Phi_{1}\right)^{2}+\lambda_{2}\left(\Phi_{2}^{\dagger} \Phi_{2}\right)^{2}+\lambda_{3}\left(\Phi_{1}^{\dagger} \Phi_{1}\right)\left(\Phi_{2}^{\dagger} \Phi_{2}\right) \\
& +\lambda_{4}\left(\Phi_{1}^{\dagger} \Phi_{2}\right)\left(\Phi_{2}^{\dagger} \Phi_{1}\right)+\frac{\lambda_{5}}{2}\left(\Phi_{1}^{\dagger} \Phi_{2}\right)^{2}+\frac{\lambda_{5}^{*}}{2}\left(\Phi_{2}^{\dagger} \Phi_{1}\right)^{2} \\
& +\lambda_{6}\left(\Phi_{1}^{\dagger} \Phi_{1}\right)\left(\Phi_{1}^{\dagger} \Phi_{2}\right)+\lambda_{6}^{*}\left(\Phi_{1}^{\dagger} \Phi_{1}\right)\left(\Phi_{2}^{\dagger} \Phi_{1}\right) \\
& +\lambda_{7}\left(\Phi_{2}^{\dagger} \Phi_{2}\right)\left(\Phi_{1}^{\dagger} \Phi_{2}\right)+\lambda_{7}^{*}\left(\Phi_{2}^{\dagger} \Phi_{2}\right)\left(\Phi_{2}^{\dagger} \Phi_{1}\right) .
\end{aligned}
$$

Note that $V$ contains four real mass parameters, $\mu_{1}^{2}, \mu_{2}^{2}$, $\operatorname{Re} m_{12}^{2}$ and $\operatorname{Im} m_{12}^{2}$, and ten real quartic couplings, $\lambda_{1,2,3,4}$, $\operatorname{Re} \lambda_{5,6,7}$ and $\operatorname{Im} \lambda_{5,6,7}$. To account for the mechanism of EWSB, the Higgs doublets $\Phi_{1,2}$ are expressed as

$$
\begin{aligned}
& \Phi_{1}=\left(\begin{array}{c}
\phi_{1}^{+} \\
\frac{1}{\sqrt{2}}\left(v_{1}+\phi_{1}+i a_{1}\right)
\end{array}\right), \\
& \Phi_{2}=e^{i \xi}\left(\begin{array}{c}
\phi_{2}^{+} \\
\frac{1}{\sqrt{2}}\left(v_{2}+\phi_{2}+i a_{2}\right)
\end{array}\right),
\end{aligned}
$$

where $\xi$ is a $C P$-odd phase, $\phi_{1,2}\left(a_{1,2}\right)$ are $C P$-even ( $C P$-odd) scalars in the $C P$-conserving limit of the theory, and $\phi_{1,2}^{+}$are positively charged scalars in the weak basis. Focusing on the neutral scalar sector, the corresponding mass matrix in the basis $\left(a_{1}, a_{2}, \phi_{1}, \phi_{2}\right)$ takes on the symmetric form,

$$
\mathcal{M}_{N}^{2}=\left(\begin{array}{cc}
\mathcal{M}_{P}^{2} & \mathcal{M}_{P S}^{2} \\
\left(\mathcal{M}_{P S}^{2}\right)^{\top} & \mathcal{M}_{S}^{2}
\end{array}\right),
$$

where $\mathcal{M}_{P}^{2}=\left(\mathcal{M}_{P}^{2}\right)^{\top}, \mathcal{M}_{S}^{2}=\left(\mathcal{M}_{S}^{2}\right)^{\top}$ and $\mathcal{M}_{P S}^{2}$ are $2 \times 2$ matrices whose explicit form may be found in [8]. Notice that in the $C P$-conserving limit of the theory, all $C P$ violating scalar-pseudoscalar mass terms vanish, i.e. $\mathcal{M}_{P S}^{2}=0$. Then, in this $C P$-conserving limit, the $2 \times 2$ mass matrices $\mathcal{M}_{P}^{2}$ and $\mathcal{M}_{S}^{2}$ can be diagonalized separately by the $\mathrm{SO}(2)$ orthogonal transformations,

$$
\begin{aligned}
\widehat{\mathcal{M}}_{P}^{2} & =\left(\begin{array}{cc}
c_{\beta} & s_{\beta} \\
-s_{\beta} & c_{\beta}
\end{array}\right) \mathcal{M}_{P}^{2}\left(\begin{array}{cc}
c_{\beta} & -s_{\beta} \\
s_{\beta} & c_{\beta}
\end{array}\right), \\
\widehat{\mathcal{M}}_{S}^{2} & =\left(\begin{array}{cc}
c_{\alpha} & s_{\alpha} \\
-s_{\alpha} & c_{\alpha}
\end{array}\right) \mathcal{M}_{S}^{2}\left(\begin{array}{cc}
c_{\alpha} & -s_{\alpha} \\
s_{\alpha} & c_{\alpha}
\end{array}\right),
\end{aligned}
$$

with $s_{x} \equiv \sin x$ and $c_{x} \equiv \cos x$, and $x=\alpha, \beta$. Upon diagonalization, the weak states are related to the mass eigenstates through

$$
\begin{aligned}
& \left(\begin{array}{l}
a_{1} \\
a_{2}
\end{array}\right)=\left(\begin{array}{cc}
c_{\beta} & -s_{\beta} \\
s_{\beta} & c_{\beta}
\end{array}\right)\left(\begin{array}{c}
G^{0} \\
A
\end{array}\right), \\
& \left(\begin{array}{l}
\phi_{1} \\
\phi_{2}
\end{array}\right)=\left(\begin{array}{cc}
c_{\alpha} & -s_{\alpha} \\
s_{\alpha} & c_{\alpha}
\end{array}\right)\left(\begin{array}{l}
h \\
H
\end{array}\right),
\end{aligned}
$$

where $G^{0}$ is the would-be Goldstone boson associated with the longitudinal degree of polarization of the $Z$ boson, $A$ is a physical $C P$-odd scalar, $h$ is identified with the observed $C P$-even Higgs boson at the LHC, and $H$ is a new heavy $C P$-even scalar having mass $M_{H}>M_{h}{ }^{1}{ }^{1}$

According to our conventions, the SM-alignment limit of the $2 \mathrm{HDM}$ is defined as the limit $\alpha \rightarrow \beta$. As was explicitly demonstrated in [24], SM alignment is realized in the 2HDM, iff the following condition is satisfied:

$$
\begin{gathered}
\lambda_{7} t_{\beta}^{4}-\left(2 \lambda_{2}-\lambda_{345}\right) t_{\beta}^{3}+3\left(\lambda_{6}-\lambda_{7}\right) t_{\beta}^{2} \\
\quad+\left(2 \lambda_{1}-\lambda_{345}\right) t_{\beta}-\lambda_{6}=0,
\end{gathered}
$$

with $\lambda_{345} \equiv \lambda_{3}+\lambda_{4}+\lambda_{5}$, for finite values of $\tan \beta \equiv t_{\beta}$, upon implementation of the SM Higgs mass constraint [23]: $M_{h} \approx 125 \mathrm{GeV}$. Obviously, if $\lambda_{6}=0$, one simple solution to (2.6) for having SM alignment is $t_{\beta}=0$. By analogy, if $\lambda_{7}=0$, SM alignment may be achieved for infinite values of $t_{\beta}$, namely when $t_{\beta} \rightarrow \infty$.

In the so-called SM-alignment limit $\alpha \rightarrow \beta$, the $h$-boson coupling to $W^{ \pm}$and $Z$ bosons has exactly the SM strength, whereas the heavier $H$ boson does not interact with the $W^{ \pm}$ and $Z$ bosons at the tree level, i.e. it becomes partially gaugophobic. ${ }^{2}$ In fact, such a scenario is getting increasingly favorable in the light of global analyses of LHC data. Nevertheless, the SM-alignment condition (2.6) would require an unpleasant degree of fine-tuning among the quartic couplings, unless there is some symmetry that enforces it. As a first step to identify possible symmetries of SM alignment, we follow [24] and require that the condition (2.6) is fulfilled for any value of $t_{\beta}$. Imposing this constraint, we find that

\footnotetext{
${ }^{1}$ It is straightforward to extend our discussion to scenarios with an inverted hierarchy: $M_{H}<M_{h}$.

${ }^{2}$ We should clarify that the $H$ boson is not entirely gaugophobic. Although the trilinear couplings $H W^{+} W^{-}$and $H Z Z$ are absent in the SM-alignment limit, quadrilinear interactions, such as $H H W^{+} W^{-}$and $H H Z Z$, are nonzero.
} 


$$
\lambda_{1}=\lambda_{2}=\lambda_{345} / 2, \quad \lambda_{6}=\lambda_{7}=0 .
$$

It is crucial to observe here that in deriving the above constraint, no reference was made on the structure of the bilinear mass terms $\mu_{1,2}^{2}$ and $m_{12}^{2}$ of the potential $V$ in (2.1). In principle, their form is not restricted from SM-alignment considerations, as long as they lead to a $C P$-conserving theory. Their role is simply to fix the parameter $\tan \beta$ to a particular value, thus relating the weak basis $\left(\Phi_{1}, \Phi_{2}\right)$ to the Higgs (mass-eigenstate) basis.

The next step taken in [24] was to identify possible maximal symmetries of the 2HDM potential $V$ that would enforce the constraint (2.7). The complete classification of all $13 \mathrm{SU}(2)_{L}$-invariant maximal symmetries of the $2 \mathrm{HDM}$ has been presented in $[31,32]$, after extending the $\mathrm{U}(1)_{Y^{-}}$ restricted bilinear formalism of [33-37]. In this way, the following three symmetries for SM alignment have been identified $[24]^{3}$ :

(i) $\operatorname{Sp}(4): \quad \lambda_{1}=\lambda_{2}=\lambda_{3} / 2, \lambda_{4}=\lambda_{5}=\lambda_{6}=\lambda_{7}=0$,

(ii) $\mathrm{SU}(2)_{\mathrm{HF}}$ : $\lambda_{1}=\lambda_{2}=\lambda_{34} / 2, \lambda_{5}=\lambda_{6}=\lambda_{7}=0$,

(iii) $\mathrm{SU}(2)_{\mathrm{HF}} \times \mathcal{C} P$ : $\lambda_{1}=\lambda_{2}=\lambda_{345} / 2, \lambda_{6}=\lambda_{7}=0$.

We note that the unitary symplectic group $\mathrm{Sp}(4)^{4}$ is acting on the reduced four-dimensional $\boldsymbol{\Phi}$ basis, defined as $\boldsymbol{\Phi} \equiv$ $\left(\Phi_{1}, \Phi_{2}, i \sigma^{2} \Phi_{1}^{*}, i \sigma^{2} \Phi_{2}^{*}\right)^{\top}$, where $\sigma^{2}$ is the second matrix of the Pauli matrices: $\sigma^{a}=\left(\sigma^{1}, \sigma^{2}, \sigma^{3}\right)$. Hence, the group $\mathrm{Sp}(4)$ defines a larger set of custodial symmetry transformations [32]. As for the symmetries SU(2) in (ii) and $\mathrm{SO}(2)$ in (iii), they are acting on the two-dimensional Higgs family (HF) space: $\left(\Phi_{1}, \Phi_{2}\right)$. Therefore, we also denote these symmetries as $\mathrm{SU}(2)_{\mathrm{HF}}$ and $\mathrm{SO}(2)_{\mathrm{HF}}$, respectively. Finally, the discrete group $\mathcal{C P}$ in (iii) refers to the canonical $C P$ symmetry, $\quad\left(\Phi_{1}(t, \mathbf{x}), \Phi_{2}(t, \mathbf{x})\right) \rightarrow\left(\Phi_{1}^{*}(t,-\mathbf{x}), \Phi_{2}^{*}(t,-\mathbf{x})\right)$, which is tacitly assumed to apply to the classical action of the theory.

Having gained valuable insight from the above exercise, one might wonder how the three symmetries of the SM alignment stated in (2.8), $\mathrm{Sp}(4), \mathrm{SU}(2)$ and $\mathrm{SO}(2)$ would manifest themselves in an explicit construction of the $2 \mathrm{HDM}$ scalar potential $V$. To this end, we observe that there are correspondingly three symmetry structures that are relevant to SM alignment:

\footnotetext{
${ }^{3}$ With the aid of the bilinear formalism, the three symmetries in (2.8) are classified as (i) $\mathrm{SO}(5) \simeq \mathrm{Sp}(4) / Z_{2}$; (ii) $\mathrm{SO}(3) \simeq \mathrm{SU}(2)_{\mathrm{HF}} /$ $Z_{2}$; and (iii) $\mathrm{SO}(2) \times Z_{2} \simeq \mathrm{SO}(2)_{\mathrm{HF}} \times \mathcal{C} P$, e.g. according to Table II of [32].

${ }^{4}$ We remind the reader about the basic set relations of $\operatorname{Sp}(2 n)$ groups: $\mathrm{SU}(n) \subset \mathrm{Sp}(2 n) \subset \mathrm{SU}(2 n)$, with $n \geq 2$.
}

$$
\begin{aligned}
S & =\Phi_{1}^{\dagger} \Phi_{1}+\Phi_{2}^{\dagger} \Phi_{2}=\frac{1}{2} \boldsymbol{\Phi}^{\dagger} \boldsymbol{\Phi}, \\
D^{a} & =\Phi_{1}^{\dagger} \sigma^{a} \Phi_{1}+\Phi_{2}^{\dagger} \sigma^{a} \Phi_{2}, \\
T & =\Phi_{1} \Phi_{1}^{\top}+\Phi_{2} \Phi_{2}^{\top} .
\end{aligned}
$$

Under an $\mathrm{SU}(2)_{L}$ gauge transformation, $\Phi_{1,2} \rightarrow \Phi_{1,2}^{\prime}=$ $U \Phi_{1,2}$, with $U \in \mathrm{SU}(2)_{L}$, the symmetry structures $S, D^{a}$ and $T$ transform as follows:

$$
\begin{aligned}
S & \rightarrow S^{\prime}=S, \quad D^{a} \rightarrow D^{\prime a}=O^{a b} D^{b}, \\
T & \rightarrow T^{\prime}=U T U^{\top},
\end{aligned}
$$

where $O \in \mathrm{SO}(3)$. Evidently, $S$ is a gauge-invariant scalar, $D^{a}$ transforms as a three-dimensional Euclidean vector and $T$ transforms as a bidoublet. Under Higgs-doublet field transformations, the quantity $S$ is an $\mathrm{Sp}(4)$ invariant in the $\boldsymbol{\Phi}$-space, while $D^{a}$ and $T$ are invariant under $\mathrm{SU}(2)$ and $\operatorname{SO}(2)$ rotations in the HF space $\left(\Phi_{1}, \Phi_{2}\right)$, respectively.

In terms of $S, D^{a}$ and $T$, the most general scalar 2HDM potential $V$ realizing natural alignment may alternatively be written down as follows,

$$
V=V_{\mathrm{sym}}+\Delta V
$$

where

$$
\begin{aligned}
V_{\text {sym }}= & -\mu^{2} S+\lambda_{S} S^{2}+\lambda_{D} D^{a} D^{a}+\lambda_{T} \operatorname{Tr}\left(T T^{*}\right) \\
= & -\mu^{2}\left(\Phi_{1}^{\dagger} \Phi_{1}+\Phi_{2}^{\dagger} \Phi_{2}\right)+\left(\lambda_{S}+\lambda_{D}+\lambda_{T}\right) \\
& \times\left[\left(\Phi_{1}^{\dagger} \Phi_{1}\right)^{2}+\left(\Phi_{2}^{\dagger} \Phi_{2}\right)^{2}\right]+2\left(\lambda_{S}-\lambda_{D}\right)\left(\Phi_{1}^{\dagger} \Phi_{1}\right)\left(\Phi_{2}^{\dagger} \Phi_{2}\right) \\
& +4 \lambda_{D}\left(\Phi_{1}^{\dagger} \Phi_{2}\right)\left(\Phi_{2}^{\dagger} \Phi_{1}\right)+\lambda_{T}\left[\left(\Phi_{1}^{\dagger} \Phi_{2}\right)^{2}+\left(\Phi_{2}^{\dagger} \Phi_{1}\right)^{2}\right],
\end{aligned}
$$

with $\mu^{2}>0,{ }^{5}$ and

$$
\Delta V=\sum_{i, j=1,2} m_{i j}^{2} \Phi_{i}^{\dagger} \Phi_{j}
$$

are soft-symmetry breaking terms. In arriving at the last equality in (2.12), we have employed the property of the Pauli matrices: $\left(\Phi_{1}^{\dagger} \sigma^{a} \Phi_{1}\right)\left(\Phi_{2}^{\dagger} \sigma^{a} \Phi_{2}\right)=2\left(\Phi_{1}^{\dagger} \Phi_{2}\right)\left(\Phi_{2}^{\dagger} \Phi_{1}\right)-$ $\left(\Phi_{1}^{\dagger} \Phi_{1}\right)\left(\Phi_{2}^{\dagger} \Phi_{2}\right)$. Obviously, the so-called maximally symmetric 2HDM studied in detail in [24], which corresponds to scenario (i) of (2.8), is obtained when $\lambda_{D}=\lambda_{T}=0$, while the $\mathrm{SU}(2)_{\mathrm{HF}^{-}}$-symmetric $2 \mathrm{HDM}$ [scenario (ii) of (2.8)] is recovered when $\lambda_{T}=0$. Finally, scenario (iii) of (2.8) is realized, if all three quartic couplings $\lambda_{S, D, T}$ in (2.12) are nonzero.

\footnotetext{
${ }^{5}$ In addition to the condition for EWSB, convexity of $V$ at large values of $\Phi_{1.2}$ in any field direction can be simply enforced by demanding that $\lambda_{S, D, T} \geq 0$.
} 
We now notice that the SM alignment symmetries given in (2.8) can be used to diagonalize the dimension-2 part of the 2 HDM potential $V$ in the HF space $\left(\Phi_{1}, \Phi_{2}\right)$. This means that the Hermitian bilinear mass matrix, which we define in the HF space as $M_{i j}^{2} \equiv-\mu^{2} \delta_{i j}+m_{i j}^{2}$, can be brought into the diagonal form $\hat{M}_{i j}^{2}$ by means of a $\mathrm{SU}(2)$ transformation, without altering the dimension-4 part of $V$, i.e. $V_{\text {sym }}$ given in (2.12). For scenario (iii), the bilinear mass matrix $M_{i j}^{2}$ must be real to be diagonalizable by means of an $\mathrm{SO}(2)$ rotation, i.e. $M_{i j}^{2}=M_{i j}^{2}$. In this HF basis, say $\left(\Phi_{1}^{\prime}, \Phi_{2}^{\prime}\right)$, in which $M_{i j}^{2}$ is diagonal, only one Higgs doublet acquires nonzero VEV, e.g. $\Phi_{1}^{\prime}$, which is identified with the VEV $v$ of the SM Higgs doublet field $\Phi$, i.e. $\left\langle\Phi_{1}^{\prime}\right\rangle \equiv\langle\Phi\rangle=v / \sqrt{2}$. This specific HF basis, i.e. $\left(\Phi_{1}^{\prime}, \Phi_{2}^{\prime}\right)$, is also called the Higgs basis [16]. Hence, the key observation is that SM-alignment-symmetry transformations not only leave the quartic couplings invariant, but also include transformations that lead to the Higgs basis. Notice that in the Higgs basis, an exact canonical $Z_{2}$ symmetry for the 2HDM potential becomes manifest, in which $\Phi_{1}^{\prime} \rightarrow+\Phi_{1}^{\prime}$ and $\Phi_{2}^{\prime} \rightarrow-\Phi_{2}^{\prime}$, which remains unbroken, even after EWSB, i.e. $\Phi_{2}^{\prime}$ becomes an inert doublet (see also our discussion below for the inert 2HDM). This symmetrybased approach proves very useful in deriving the complete set of symmetries for alignment in $n \mathrm{HDMs}$, with $n>2$, in the next section.

Besides the softly broken SM-alignment symmetries stated in (2.8), under which the EWSB Higgs doublets $\Phi_{1}$ and $\Phi_{2}$ have nontrivial transformation properties, another possible way for getting natural alignment in $2 \mathrm{HDM}$ is to impose an unbroken discrete $Z_{2}$ symmetry $n$, under which one of the Higgs doublets is $Z_{2}$ odd, e.g. $\Phi_{2} \rightarrow-\Phi_{2}$. In this class of scenarios, in which $\lambda_{6}=\lambda_{7}=0$ and $m_{12}^{2}=0$, while the quartic couplings $\lambda_{1,2,3,4,5}$ are unconstrained, the Higgs basis is fixed to be along the $Z_{2}$-even scalar field, e.g. $\Phi_{1}$, representing the SM Higgs doublet $\Phi$. Such naturally aligned scenarios, in which the $Z_{2}$-odd Higgs doublet is an inert field, correspond to the limits $t_{\beta} \rightarrow 0$ (for $\Phi_{1} \equiv \Phi$ ) or $t_{\beta} \rightarrow \infty$ (for $\left.\Phi_{2} \equiv \Phi\right)$. As we see in the next section, the inert scalar sector in multi-HDMs can have much richer structure possessing its own set of symmetries.

\section{NATURAL ALIGNMENT IN MULTI-HDMS}

It is straightforward to generalize the results of the previous section and derive the complete set of symmetries for alignment in $n \mathrm{HDMs}$, with $n>2$. We may assume that the scalar sector of the theory consists of $m$ inert Higgs doublets $\widehat{\Phi}_{\hat{a}}$ (with $\left.\hat{a}=\hat{1}, \hat{2}, \ldots, \widehat{m}\right)$, for which $\left\langle\widehat{\Phi}_{\hat{a}}\right\rangle=0$, and $N_{H} \equiv n-m$ Higgs doublets $\Phi_{a}$ (with $a=1,2, \ldots, N_{H}$ ) which generally take part in EWSB with nonzero VEVs, i.e. $\left\langle\Phi_{a}\right\rangle \neq 0$. The vanishing of the VEVs of the inert Higgs doublets is enforced by imposing a suitable discrete or continuous symmetry $\mathcal{D}$, which remains unbroken after EWSB. Under the action of $\mathcal{D}$, the noninert $N_{H}$ Higgs doublets $\Phi_{a}$ transform trivially, i.e. $\Phi_{a} \rightarrow \Phi_{a}$. In general, the discrete group $\mathcal{D}$ could be $Z_{2}$, or even a higher $Z_{N}$ symmetry, but not the canonical $\mathcal{C} P$ and/or a permutation symmetry, such as $S_{3}$, since such symmetries do not necessarily imply the vanishing of the VEVs for the inert Higgs doublets $\widehat{\Phi}_{\hat{a}}$.

The full scalar potential $V$ of a naturally aligned $n \mathrm{HDM}$ may be written down as a sum of three terms,

$$
V=V_{\text {sym }}+V_{\text {inert }}+\Delta V,
$$

where $V_{\text {sym }}$ describes the symmetry-constrained part of the scalar sector, which is responsible for the EWSB of the theory. Its form turns out to be the same as the one found in the 2HDM [cf. (2.12)], which we elucidate in more detail below. In addition, the potential term $V_{\text {inert }}$ in (3.1) represents the inert scalar sector which does not participate in EWSB, i.e.

$$
\begin{aligned}
V_{\text {inert }}= & \widehat{m}_{\hat{a} \hat{b}}^{2} \widehat{\Phi}_{\hat{a}}^{\dagger} \widehat{\Phi}_{\hat{b}}+\lambda_{\hat{a} \hat{b} \hat{c} \hat{d}}\left(\widehat{\Phi}_{\hat{a}}^{\dagger} \widehat{\Phi}_{\hat{b}}\right)\left(\widehat{\Phi}_{\hat{c}}^{\dagger} \widehat{\Phi}_{\hat{d}}\right) \\
& +\lambda_{\hat{a} \hat{b} c d}\left(\widehat{\Phi}_{\hat{a}}^{\dagger} \widehat{\Phi}_{\hat{b}}\right)\left(\Phi_{c}^{\dagger} \Phi_{d}\right)+\lambda_{a \hat{b} \hat{c} d}\left(\Phi_{a}^{\dagger} \widehat{\Phi}_{\hat{b}}\right)\left(\widehat{\Phi}_{\hat{c}}^{\dagger} \Phi_{d}\right) \\
& +\left[\lambda_{a \hat{b} c \hat{d}}\left(\Phi_{a}^{\dagger} \widehat{\Phi}_{\hat{b}}\right)\left(\Phi_{c}^{\dagger} \widehat{\Phi}_{\hat{d}}\right)+\text { H.c. }\right],
\end{aligned}
$$

where summation of the indices over their allowed range of values is understood, and $\lambda_{\hat{a} \hat{b} \hat{c} \hat{d}}, \lambda_{\hat{a} \hat{b} c d}, \lambda_{a \hat{b} \hat{b} d}$ and $\lambda_{\hat{a} b \hat{c} d}$ are all different sets of quartic couplings. The $m \times m$ matrix $\widehat{m}_{\hat{a} \hat{b}}^{2}$ is taken to be positive definite, so as to avoid spontaneous EWSB. From the explicit construction of the inert scalar sector in (3.2), we readily see that it remains invariant under the $Z_{2}$ symmetry,

$$
Z_{2}^{\mathrm{I}}: \Phi_{a} \rightarrow \Phi_{a}, \quad \widehat{\Phi}_{\hat{b}} \rightarrow-\widehat{\Phi}_{\hat{b}} .
$$

Consequently, $Z_{2}^{\mathrm{I}}$ should always be contained in the $\mathcal{D}$ symmetry group of the inert scalar sector, i.e. $Z_{2}^{\mathrm{I}} \subset \mathcal{D}$. Finally, the third term $\Delta V$ in (3.1) contains the softsymmetry breaking mass parameters of the EWSB sector and is given by

$$
\Delta V=m_{a b}^{2} \Phi_{a}^{\dagger} \Phi_{b},
$$

where $m_{a b}^{2}$ is in general a Hermitian $N_{H} \times N_{H}$ matrix. Note that dimension-2 mixed bilinear operators, such as $\widehat{\Phi}_{\hat{a}}^{\dagger} \Phi_{b}$, are not allowed in the theory.

On the basis of the assumption that the soft-symmetry breaking mass matrix $m_{a b}^{2}$ in (3.4) has no particular discrete symmetry structure, ${ }^{6}$ there are then three possible

\footnotetext{
${ }^{6}$ Our assumption that $m_{a b}^{2}$ must have no particular discrete symmetry structure may also be motivated by the strong constraints on the nonobservation of domain walls [31] that are produced from the spontaneous breaking of possible ungauged discrete symmetries in the theory at the SM electroweak phase transition.
} 
continuous symmetry groups that can bring $m_{a b}^{2}$ into the diagonal form, as required to happen in the Higgs basis. These three continuous maximal symmetries for SM alignment, which should be respected by the EWSB potential term $V_{\text {sym }}$ in (3.1), are the generalization of those found in the $2 \mathrm{HDM}$, i.e.

$$
\begin{aligned}
& \text { (i) } \operatorname{Sp}\left(2 N_{H}\right) \times \mathcal{D}, \\
& \text { (ii) } \operatorname{SU}\left(N_{H}\right) \times \mathcal{D}, \\
& \text { (iii) } \mathrm{SO}\left(N_{H}\right) \times \mathcal{C P} \times \mathcal{D},
\end{aligned}
$$

where the discrete $\mathcal{C P}$ symmetry is acting on the noninert scalar sector consisting of $N_{H}=n-m$ Higgs doublets $\Phi_{a}$, with $N_{H}>1$. We note that, for $N_{H}=1$, the EWSB part of the potential $V_{\text {sym }}$ becomes identical to the SM potential, whereas the symmetry group $\mathcal{D}$ of the inert sector may have its own rich structure, as we see below. As mentioned in Sec. II, we emphasize again that for the maximal symmetry (iii), the softsymmetry breaking mass matrix $m_{a b}^{2}$ must be real or close to real, in order to avoid too large tree-level $C P$-violating scalarpseudoscalar transitions, whose effects are severely constrained by limits on electric dipole moments $[38,39]$.

We note that as in the 2HDM, the effect of $\Delta V$ given in (3.4) is to fix the rotation angles of the continuous symmetries in (3.5) to particular values that relate the weak-basis fields $\Phi_{a}$ to the corresponding fields $\Phi_{a}^{\prime}$ in the Higgs (mass-eigenstate) basis. In the Higgs basis, one of the rotated scalar doublets, e.g. $\Phi_{1}^{\prime}$, becomes aligned to the SM Higgs doublet $\Phi$, i.e. $\Phi_{1}^{\prime} \equiv \Phi$, such that $\left\langle\Phi_{1}^{\prime}\right\rangle=\langle\Phi\rangle=$ $v / \sqrt{2}$ is the SM VEV. Like in the 2HDM case, in the Higgs basis, an exact canonical $Z_{2}$ symmetry for the EWSB part of the $n \mathrm{HDM}$ potential becomes manifest,

$$
Z_{2}^{\mathrm{EW}}: \Phi_{1}^{\prime} \rightarrow \Phi_{1}^{\prime}, \quad \Phi_{a^{\prime}}^{\prime} \rightarrow-\Phi_{a^{\prime}}^{\prime},
$$

where $a^{\prime}=2,3, \ldots, N_{H}$. As a consequence, after EWSB, the full $n$ HDM potential becomes invariant under $Z_{2}^{\mathrm{EW}} \times Z_{2}^{\mathrm{I}}$, where $Z_{2}^{\mathrm{I}}$ is given in (3.3). In fact, this residual product symmetry $Z_{2}^{\mathrm{EW}} \times Z_{2}^{\mathrm{I}}$ is instrumental, as it is the one that enforces SM alignment in the $n \mathrm{HDM}$, even beyond the tree-level approximation.

Let us now turn our attention to the symmetryconstrained part of the scalar sector $V_{\text {sym }}$ that occurs in the $n \mathrm{HDM}$ potential $V$ in (3.1). We demonstrate that its analytical form is the same as the one found in the 2HDM. To elucidate this point, we first introduce the symmetrycovariant structures,

$$
\begin{aligned}
S_{\mathcal{A}} & =\sum_{k, l=1}^{N_{H}} \Phi_{k}^{\dagger}\left[\mathcal{A}_{k l} \otimes \mathbf{1}_{2}\right] \Phi_{l}, \quad D_{\mathcal{B}}^{a}=\sum_{k, l=1}^{N_{H}} \Phi_{k}^{\dagger}\left[\mathcal{B}_{k l} \otimes \sigma^{a}\right] \Phi_{l}, \\
T_{\mathcal{C}} & =\sum_{k, l=1}^{N_{H}} \Phi_{k} \mathcal{C}_{k l} \Phi_{l}^{\top},
\end{aligned}
$$

where $\mathcal{A}=\mathcal{A}^{\dagger}, \mathcal{B}=\mathcal{B}^{\dagger}$ and $\mathcal{C}= \pm \mathcal{C}^{\top}$ are all $N_{H} \times N_{H}$ matrices. Observe that the three symmetry structures $S_{\mathcal{A}}$, $D_{\mathcal{B}}^{a}$ and $T_{\mathcal{C}}$ all share the same transformation properties under the $\mathrm{SU}(2)_{L}$ gauge group as the corresponding ones given in (2.10) for the 2HDM. Specifically, $S_{\mathcal{A}}$ is a gaugeinvariant singlet, $D_{\mathcal{B}}^{a}$ transforms as a three-dimensional Euclidean vector, and $T_{\mathcal{C}}$ transforms as a bidoublet. In principle, one could have also considered more involved covariant objects of the form $D_{\mathcal{B}}^{a b \ldots}=\sum_{k, l=1}^{n-m} \Phi_{k}^{\dagger}\left[\mathcal{B}_{k l} \otimes\right.$ $\left.\left(\sigma^{a} \sigma^{b} \ldots\right)\right] \Phi_{l}$. Then, gauge-invariant objects, such as $\left(D_{\mathcal{B}}^{a b \ldots}\right)^{2}$, which would potentially contribute to $V_{\text {sym }}$, can always be reduced to $\left(S_{\mathcal{A}}\right)^{2}$ and $\left(D_{\mathcal{B}}^{a}\right)^{2}$, through the successive use of the identities $\sigma^{a} \sigma^{b}=\mathbf{1}_{2} \delta^{a b}+i \epsilon^{a b c} \sigma^{c}$ and $\epsilon^{a b c} \epsilon^{a b d}=2 \delta^{c d}$. Hence, such higher rank tensors, under the $\mathrm{SU}(2)_{L}$ gauge group, do not introduce new candidate structures for $V_{\text {sym }}$, other than the ones stated in (3.7).

Let us now consider symmetry transformations in the HF space of the EWSB sector. Under such transformations, the structures $S_{\mathcal{A}}, D_{\mathcal{B}}^{a}$ and $T_{\mathcal{C}}$ stated in (3.7) must transform covariantly with respect to $\mathrm{SU}\left(N_{H}\right) \subset \mathrm{Sp}\left(2 N_{H}\right)$ and $\mathrm{SO}\left(N_{H}\right)$ global groups. This means that the $N_{H} \times N_{H}$ matrices $\mathcal{A}, \mathcal{B}$ and $\mathcal{C}$ must be products of the generators $T^{a}$ of either $\mathrm{SU}\left(N_{H}\right)$ or $\mathrm{SO}\left(N_{H}\right)$ groups (including the identity $\mathbf{1}_{N_{H}}$ ), in the fundamental representation. These products of $T^{a}$ generate tensors in the group space and may also have particular symmetry orderings as determined by the Young tableaux. For instance, nontrivial rank-2 tensor objects can be produced, if $\mathcal{A}, \mathcal{B}, \mathcal{C}=-i\left[T^{a}, T^{b}\right]=f^{a b c} T^{c}$, or if $\mathcal{A}, \mathcal{B}, \mathcal{C}=\left\{T^{a}, T^{b}\right\} \propto d^{a b c} T^{c}$, for $\operatorname{SU}\left(N_{H}>2\right)$ and $\mathrm{SO}\left(N_{H}>4\right)$. However, taking into account the known identities for the groups generators,

$\mathrm{SU}\left(N_{H}\right):\left(T^{a}\right)_{i j}\left(T^{a}\right)_{k l}=\frac{1}{2}\left(\delta_{i l} \delta_{k j}-\frac{1}{N_{H}} \delta_{i j} \delta_{k l}\right)$,

$\mathrm{SO}\left(N_{H}\right):\left(T^{a}\right)_{i j}\left(T^{a}\right)_{k l}=2\left(\delta_{i l} \delta_{k j}-\delta_{i j} \delta_{k l}\right)$,

and the fact that $f^{a b c} f^{a b d}, d^{a b c} d^{a b d} \propto \delta^{c d}$, it is then not difficult to show that all symmetry-invariant objects of potential interest, such as $\left(S_{\mathcal{A}}\right)^{2},\left(D_{\mathcal{B}}^{a}\right)^{2}$ and $\operatorname{Tr}\left(T_{\mathcal{C}} T_{\mathcal{C}}^{*}\right)$, reduce to linear combinations of the objects $\left(S_{1}\right)^{2},\left(D_{1}^{a}\right)^{2}$ and $\operatorname{Tr}\left(T_{\mathbf{1}} T_{\mathbf{1}}^{*}\right)$, with $\mathcal{A}=\mathcal{B}=\mathcal{C}=\mathbf{1}_{N_{H}}$ in (3.7). Therefore, the HF-singlet structures, $S_{1}, D_{1}^{a}$ and $T_{1}$, are sufficient to describe the symmetry-constrained part of the scalar sector $V_{\text {sym }}$, i.e.

$$
V_{\text {sym }}=-\mu^{2} S_{1}+\lambda_{S} S_{1}^{2}+\lambda_{D} D_{1}^{a} D_{1}^{a}+\lambda_{T} \operatorname{Tr}\left(T_{1} T_{1}^{*}\right),
$$

with $\mu^{2}>0$. Consequently, the general group-structure form of $V_{\text {sym }}$ in $n \mathrm{HDMs}$ (with $n>2$ ) is the same as the one found in the 2HDM [cf. (2.12)].

For illustration, let us now apply our findings in (3.5), in order to obtain all the admissible forms for naturally aligned $2 \mathrm{HDM}$ and $3 \mathrm{HDM}$ potentials. In the $2 \mathrm{HDM}$, if 
both the Higgs doublets $\Phi_{1,2}$ participate in EWSB, (3.5) leads obviously to the three maximal symmetry groups presented in Sec. II: (i) $\mathrm{Sp}$ (4); (ii) $\mathrm{SU}(2)$; (iii) $\mathrm{SO}(2) \times \mathcal{C} P$ [cf. (2.8)]. Now, if one of the scalar doublets of the $2 \mathrm{HDM}$ is inert, e.g. $\widehat{\Phi}_{\hat{2}}(m=1)$, then there are three distinct possibilities:

(i) the $Z_{2}=Z_{2}^{\mathrm{I}}$ symmetry [27-30] as discussed in the previous section [cf. (3.3)];

(ii) the unbroken Peccei-Quinn-type U(1) symmetry [40,41], enforcing that $\lambda_{12} 1 \hat{2} \equiv \lambda_{5}=0$, under which $\widehat{\Phi}_{\hat{2}}$ is charged;

(iii) the custodial symmetry $\mathrm{Sp}(2) \simeq \mathrm{SU}(2)_{C . \text { I. }}$ acting on the inert field space $\left(\widehat{\Phi}_{\hat{2}}, i \sigma^{2} \widehat{\Phi}_{\hat{2}}^{*}\right)$, which implies that $\lambda_{1 \hat{2} \hat{1} \hat{2}} \equiv \lambda_{5}=0$ and $\lambda_{1 \hat{2} \hat{2} 1} \equiv \lambda_{4}=0$. This symmetry is classified as $\mathrm{SO}(4) \simeq \mathrm{SU}(2)_{C, \mathrm{EW}} \times \mathrm{SU}(2)_{\mathrm{C}, \mathrm{I}}$ in Table I (number 11) of [32], where the first custodial group $\mathrm{SU}(2)_{C, \mathrm{EW}}$ acts on the EWSB sector.

We now turn our attention to the 3HDM. If all three Higgs doublets participate in the mechanism of EWSB which corresponds to $m=0$, the symmetries of alignment resulting from (3.5) are

$$
\text { (i) } \mathrm{Sp}(6), \quad \text { (ii) } \mathrm{SU}(3), \quad \text { (iii) } \mathrm{SO}(3) \times \mathcal{C} P \text {, }
$$

where $\mathrm{Sp}(6)$ is acting on the six-dimensional $\boldsymbol{\Phi}$-multiplet, $\boldsymbol{\Phi} \equiv\left(\Phi_{1}, \Phi_{2}, \Phi_{3}, i \sigma^{2} \Phi_{1}^{*}, i \sigma^{2} \Phi_{2}^{*}, i \sigma^{2} \Phi_{3}^{*}\right)^{\top}$, and $\operatorname{SU}(3)$ and $\operatorname{SO}(3)$ on the HF space: $\left(\Phi_{1}, \Phi_{2}, \Phi_{3}\right)$. This means that the $3 \mathrm{HDM}$ potential has the symmetry form of (3.10), softly broken by terms as given in (3.4).

Considering now the case of one inert Higgs doublet, e.g. $\widehat{\Phi}_{\hat{3}}(m=1)$, the symmetries of the inert sector are (i) the $Z_{2}$ symmetry, with $\widehat{\Phi}_{\hat{3}} \rightarrow-\widehat{\Phi}_{\hat{3}}$; (ii) the global U(1) symmetry, under which $\widehat{\Phi}_{\hat{3}}$ carries nonzero charge, leading to $\lambda_{a \hat{3} b \hat{3}}=$ 0 (with $a, b=1,2$ ); and (iii) the custodial symmetry group $\mathrm{Sp}(2) \simeq \mathrm{SU}(2)_{C}$ acting on the field space $\left(\widehat{\Phi}_{\hat{3}}, i \sigma^{2} \widehat{\Phi}_{\hat{3}}^{*}\right)$, which implies that $\lambda_{a \hat{3} \hat{3} b}=0$ and $\lambda_{a \hat{3} b \hat{3}}=0$.

The last possible class of naturally aligned $3 \mathrm{HDMs}$ is the one that has two inert Higgs doublets, $\widehat{\Phi}_{\hat{2}}$ and $\widehat{\Phi}_{\hat{3}}(m=2)$. In addition to the inert $Z_{2}$ symmetry in (3.3), $\Phi_{1} \rightarrow \Phi_{1}$, $\widehat{\Phi}_{\hat{2}} \rightarrow-\widehat{\Phi}_{\hat{2}}$ and $\widehat{\Phi}_{\hat{3}} \rightarrow-\widehat{\Phi}_{\hat{3}}$, one inherits at least all 13 maximal symmetries of the 2HDM [31,32] when $\lambda_{1 \hat{a} \hat{b} 1}=$ $\lambda_{1 \hat{a} 1 \hat{b}}=0$ (with $\hat{a}, \hat{b}=\hat{2}, \hat{3}$ ) in (3.2), where $\operatorname{Sp}(4)$ is the largest symmetry group. However, if $\lambda_{1 \hat{a} \hat{b} 1}$ and $\lambda_{1 \hat{a} 1 \hat{b}}$ are nonzero, further symmetries, which include $Z_{2}^{\mathrm{I}}$, may exist in the 3 HDM potential that forbid $\widehat{\Phi}_{\hat{2}}$ and $\widehat{\Phi}_{\hat{3}}$ from developing a VEV. A recent example is the generalized $C P$ symmetry of order 4 observed recently in [42]. It should be stressed here again that all the symmetries of the 3HDM inert sector must remain unbroken under EWSB.

For $n \mathrm{HDMs}$ with $n>3$, the complexity of the classification of the inert scalar sector increases, but results from lower $n$-cases become crucial in our approach to constructing all naturally aligned multi-HDMs. Instead, the alignment symmetries for the noninert sector are fully specified by the three maximal symmetries given in (3.5). If other inert scalars are present in the theory, e.g. singlets $S_{i}$ or triplets $\Delta_{l}$, our results still hold true for the EWSB part (3.10) of the scalar potential. Thus, the approach presented here is not confined to multi-HDMs only, but it can easily be generalized to more abstract scalar sectors.

\section{CONCLUSIONS}

We have derived the complete set of maximal symmetries for SM alignment that could take place in the treelevel scalar potential of the SM, with $n \geq 2$ Higgs doublets. Our results generalize the symmetries of SM alignment, previously obtained in the context of two-Higgs doublet models (the $n=2$ case [24]), without decoupling of large mass scales or recourse to specific model parameter arrangements. For the scalar sector participating in the EWSB mechanism, the general symmetry conditions for natural SM alignment are given by Eq. (3.5), which is one of the central results of this paper.

Another highlight of our study is that the inert scalar sector of multi-HDMs may have its own rich symmetry structure. In particular, within our symmetry-based approach, we have found that the 2HDM with one inert Higgs doublet may exhibit three distinct maximal symmetries: (i) the discrete $Z_{2}=Z_{2}^{\mathrm{I}}$ symmetry [27-30]; (ii) the $\mathrm{U}(1)$ symmetry and (iii) the custodial symmetry $\mathrm{SU}(2)_{C, \mathrm{I}}$, which all remain unbroken after EWSB. In addition to the frequently considered symmetry (i), it would be interesting to explore the phenomenological and cosmological implications of the inert symmetries (ii) and (iii).

We should stress again that the mechanism of SM alignement does not get invalidated, even to all orders, if soft-symmetry breaking masses [cf. (3.4)] are added to the theory. As noted in Sec. III [cf. (3.6)], this is because of the presence of an exact $Z_{2}^{\mathrm{EW}} \times Z_{2}^{\mathrm{I}}$ symmetry in the $n \mathrm{HDM}$ potential, which becomes manifest in the Higgs basis [16]. In particular, beyond the tree-level approximation, the alignement symmetries are preserved by the $\mathrm{SU}(2)_{L}$ gauge interactions, whereas the hypercharge $\mathrm{U}(1)_{Y}$ group only breaks, $\mathrm{Sp}(2 n) \rightarrow \mathrm{SU}(n)$, without spoiling the SM alignment. Instead, the Yukawa sector of the theory violates explicitly the alignment symmetries, and so it could sizeably break the SM alignment beyond the tree level. However, such explicit violations are highly model dependent, and for a given flavor structure of the Yukawa sector, they lead to predictable deviations [24] from the SM values of the Higgs-boson couplings to $W^{ \pm}$and $Z$ bosons that may constrain the parameter space of the theory. In this context, it is useful to remark that the effect of these constraints can be drastically reduced in the so-called Yukawa-aligned models [43], if the quark-Yukawa basis happens to be coincidentally aligned with the Higgs basis of the $n$ HDM. In conclusion, given the existing strict limits on non-SM 
deviations in the Higgs couplings to $W^{ \pm}$and $Z$ bosons, Eq. (3.5) provides an important constraint on future model building of multi-HDMs predicting additional low-scale scalars with masses being in the explorable sub-TeV range of the LHC.

\section{ACKNOWLEDGMENTS}

This work is supported in part by the LancasterManchester-Sheffield Consortium for Fundamental Physics, under STFC research Grant No. ST/L000520/1.
[1] The ATLAS Collaboration, Constraints on new phenomena via Higgs coupling measurements with the ATLAS detector, Report No. ATLAS-CONF-2014-010.

[2] V. Khachatryan et al. (CMS Collaboration), Searches for heavy Higgs bosons in two-Higgs-doublet models and for $t \rightarrow c h$ decay using multilepton and diphoton final states in $p p$ collisions at $8 \mathrm{TeV}$, Phys. Rev. D 90, 112013 (2014).

[3] K. Cheung, J. S. Lee, and P. Y. Tseng, Higgs precision analysis updates 2014, Phys. Rev. D 90, 095009 (2014).

[4] D. Chowdhury and O. Eberhardt, Global fits of the two-loop renormalized two-Higgs doublet model with soft $\mathrm{Z}_{2}$ breaking, J. High Energy Phys. 11 (2015) 052.

[5] N. Craig, F. D'Eramo, P. Draper, S. Thomas, and H. Zhang, The hunt for the rest of the Higgs bosons, J. High Energy Phys. 06 (2015) 137.

[6] K. A. Olive (Particle Data Group Collaboration), Review of particle physics, Chin. Phys. C 38, 090001 (2014).

[7] T. D. Lee, A theory of spontaneous T violation, Phys. Rev. D 8, 1226 (1973).

[8] A. Pilaftsis and C.E. M. Wagner, Higgs bosons in the minimal supersymmetric standard model with explicit $C P$ violation, Nucl. Phys. B553, 3 (1999).

[9] For a comprehensive review on $2 \mathrm{HDMs}$, see G. C. Branco, P. M. Ferreira, L. Lavoura, M. N. Rebelo, M. Sher, and J. P. Silva, Theory and phenomenology of two-Higgs doublet models, Phys. Rep. 516, 1 (2012).

[10] S. Weinberg, Gauge Theory of $C P$ Violation, Phys. Rev. Lett. 37, 657 (1976).

[11] B. Grzadkowski, O. M. Ogreid, P. Osland, A. Pukhov, and M. Purmohammadi, Exploring the $C P$-violating inert-doublet model, J. High Energy Phys. 06 (2011) 003.

[12] R. Gonzlez Felipe, I. P. Ivanov, C. C. Nishi, H. Serdio, and J. P. Silva, Constraining multi-Higgs flavor models, Eur. Phys. J. C 74, 2953 (2014).

[13] V. Keus, S. F. King, and S. Moretti, Phenomenology of the inert $(2+1)$ and $(4+2)$ Higgs doublet models, Phys. Rev. D 90, 075015 (2014).

[14] G. C. Branco and I. P. Ivanov, Group-theoretic restrictions on generation of $C P$ violation in multi-Higgs doublet models, J. High Energy Phys. 01 (2016) 116.

[15] D. Emmanuel-Costa, O. M. Ogreid, P. Osland, and M. N. Rebelo, Spontaneous symmetry breaking in the $S_{3}$-symmetric scalar sector, J. High Energy Phys. 02 (2016) 154.

[16] H. Georgi and D. V. Nanopoulos, Suppression of flavor changing effects from neutral spinless meson exchange in gauge theories, Phys. Lett. 82B, 95 (1979).
[17] J.F. Gunion and H.E. Haber, The $C P$-conserving twoHiggs doublet model: the approach to the decoupling limit, Phys. Rev. D 67, 075019 (2003).

[18] I. F. Ginzburg and M. Krawczyk, Symmetries of two-Higgs doublet model and CP violation, Phys. Rev. D 72, 115013 (2005).

[19] I. F. Ginzburg, M. Krawczyk, and P. Osland, in Proceedings of the International Workshop on Linear Colliders, edited by E. Fernandez and A. Pacheco (UAB, Sitges, Spain, 1999).

[20] P. H. Chankowski, T. Farris, B. Grzadkowski, J. F. Gunion, J. Kalinowski, and M. Krawczyk, Do precision electroweak constraints guarantee $e^{+} e^{-}$collider discovery of at least one Higgs boson of a two-Higgs doublet model? Phys. Lett. B 496, 195 (2000).

[21] I. F. Ginzburg, M. Krawczyk, and P. Osland, LC Note No. LC-TH-2001-026; Potential of Photon Collider in resolving SM-like scenarios, Nucl. Instrum. Methods Phys. Res., Sect. A 472, 149 (2001); in Physics and Experiments with Future Linear $e^{+} e^{-}$Colliders, AIP Conf. Proc. No. 578 (AIP, New York, 2001), pp. 304-311.

[22] A. Delgado, G. Nardini, and M. Quiros, A light supersymmetric Higgs sector hidden by a standard model-like Higgs, J. High Energy Phys. 07 (2013) 054.

[23] M. Carena, I. Low, N. R. Shah, and C. E. M. Wagner, Impersonating the standard model Higgs boson: alignment without decoupling, J. High Energy Phys. 04 (2014) 015.

[24] P. S. Bhupal Dev and A. Pilaftsis, Maximally symmetric two-Higgs doublet model with natural standard model alignment, J. High Energy Phys. 12 (2014) 024; P. S. Bhupal Dev and A. Pilaftsis, J. High Energy Phys. 11 (2015) 147(E).

[25] J. Bernon, J. F. Gunion, H. E. Haber, Y. Jiang, and S. Kraml, Scrutinizing the alignment limit in two-Higgs doublet models: $m_{h}=125 \mathrm{GeV}$, Phys. Rev. D 92, 075004 (2015).

[26] J. Bernon, J. F. Gunion, H. E. Haber, Y. Jiang, and S. Kraml, Scrutinizing the alignment limit in two-Higgs doublet models. Part 2: $m_{H}=125 \mathrm{GeV}$, Phys. Rev. D 93, 035027 (2016).

[27] S. L. Glashow and S. Weinberg, Natural conservation laws for neutral currents, Phys. Rev. D 15, 1958 (1977).

[28] N. G. Deshpande and E. Ma, Pattern of symmetry breaking with two-Higgs doublets, Phys. Rev. D 18, 2574 (1978).

[29] V. Silveira and A. Zee, Scalar phantoms, Phys. Lett. B 161, 136 (1985). 
[30] R. Barbieri, L. J. Hall, and V.S. Rychkov, Improved naturalness with a heavy Higgs: an alternative road to LHC physics, Phys. Rev. D 74, 015007 (2006).

[31] R. A. Battye, G. D. Brawn, and A. Pilaftsis, Vacuum topology of the two-Higgs doublet model, J. High Energy Phys. 08 (2011) 020.

[32] A. Pilaftsis, On the classification of accidental symmetries of the two-Higgs doublet model potential, Phys. Lett. B 706, 465 (2012).

[33] M. Maniatis, A. von Manteuffel, and O. Nachtmann, $C P$ violation in the general two-Higgs doublet model: a geometric view, Eur. Phys. J. C 57, 719 (2008).

[34] I. P. Ivanov, Minkowski space structure of the Higgs potential in 2HDM. II. Minima, symmetries, and topology, Phys. Rev. D 77, 015017 (2008).

[35] C. C. Nishi, Physical parameters and basis transformations in the two-Higgs doublet model, Phys. Rev. D 77, 055009 (2008).

[36] P. M. Ferreira, H. E. Haber, and J. P. Silva, Generalized $C P$ symmetries and special regions of parameter space in the two-Higgs doublet model, Phys. Rev. D 79, 116004 (2009).
[37] P. M. Ferreira, H. E. Haber, M. Maniatis, O. Nachtmann, and J.P. Silva, Geometric picture of generalized-CP and Higgs-family transformations in the two-Higgs doublet model, Int. J. Mod. Phys. A A26, 769 (2011).

[38] K. Cheung, J. S. Lee, E. Senaha, and P. Y. Tseng, Confronting Higgcision with electric dipole moments, J. High Energy Phys. 06 (2014) 149.

[39] W. Dekens, J. de Vries, J. Bsaisou, W. Bernreuther, C. Hanhart, U. G. Meiner, A. Nogga, and A. Wirzba, Unraveling models of $C P$ violation through electric dipole moments of light nuclei, J. High Energy Phys. 07 (2014) 069.

[40] R. D. Peccei and H. R. Quinn, CP Conservation in the Presence of Instantons, Phys. Rev. Lett. 38, 1440 (1977).

[41] R. D. Peccei and H. R. Quinn, Constraints imposed by $C P$ conservation in the presence of instantons, Phys. Rev. D 16, 1791 (1977).

[42] I. P. Ivanov and J. P. Silva, A CP-conserving multi-Higgs model without real basis, arXiv:1512.09276.

[43] A. Pich and P. Tuzon, Yukawa alignment in the two-Higgs doublet model, Phys. Rev. D 80, 091702 (2009). 